\title{
Exhibition of Postural Time-Force Parameters in Varying Severity of Osteoarthritis of Knee During Performing Sit-To-Stand Testing
}

\author{
Shin-Tsu Chang* 1,2,3, Hsin-Yi Wang, ${ }^{2,4}$, Shun-Hwa Wei ${ }^{4}$ and Shao-Chi Lu ${ }^{5}$ \\ ${ }^{1}$ Department of Physical Medicine and Rehabilitation, Taichung Veterans General Hospital, Taiwan \\ ${ }^{2}$ Department of Physical Medicine and Rehabilitation, Tri-Service General Hospital, School of Medicine, National Defense Medical Center, Taipei, Taiwan \\ ${ }^{3}$ School of Medicine, Chung Shan Medical University, Taichung, Taiwan \\ ${ }^{4}$ Department and Institute of Physical Therapy and Assistive Technology, National Yang-Ming University, Taipei, Taiwan \\ ${ }^{5}$ Department of Physical Medicine and Rehabilitation, Hua-Lien Armed Forces General Hospital, Hua-Lien, Taiwan \\ *Corresponding author: Shin-Tsu Chang, Department of Physical Medicine and Rehabilitation, Taichung Veterans General Hospital, \\ Tri-Service General Hospital, National Defense Medical Center, Chung Shan Medical University, Taiwan. \\ To Cite This Article: Shin-Tsu Chang. Exhibition of Postural Time-Force Parameters in Varying Severity of Osteoarthritis of Knee During \\ Performing Sit-To-Stand Testing. Am J Biomed Sci \& Res. 2019 - 4(1). AJBSR.MS.ID.000754. DOI: 10.34297/AJBSR.2019.04.000754
}

Received: July 05, 2019 | Published: July 16, 2019

\# CHANG ST and WANG HY contribute equally.

\begin{abstract}
Background: Knee osteoarthritis $(\mathrm{OA})$ is an irreversible leading cause of disability in elder. Clinical and research assessment for knee osteoarthritis often use radiographic finding to determine the disease severity. But recently research found that radiographic diagnosis couldn't reflect the functional activity of life capacity of patient with knee osteoarthritis. Load distribution influence functional capacity of patient with knee osteoarthritis. It is important to know the correlation between performance and functional capacity of knee osteoarthritis patients.
\end{abstract}

Purpose: In this study, a sit-to-stand task was chosen to compare the force-plate measured time-force parameters and weight bearing asymmetry degree in knee osteoarthritis patients with different functional severity.

Method: Study content sit-to-stand performance task and Lequesne severity index questionnaire. Forty-nine subjects participated in this study and were categorized into four groups (normal, mild functional limited OA knee patient, moderate functional limited OA knee patient, severe functional limited OA knee patient) according to Lequesne severity index questionnaire grading which assesses the functional disability of knee osteoarthritis patients. Sit-to-stand performance used a four-load cell force-plate to detect ground reaction force during movement. Then four parameters (sit-to-stand total time, the ground reaction force, the moving rate of center of force (COP), and asymmetry index) which measured by force-plate were compared with the Lequesne severity index questionnaire grading. One-way ANOVA was used to compare the four parameters difference between four groups.

Results and discussion: Compared with the normal subjects, the knee osteoarthritis patients take more time to stand up ( $p=0.003$ ). Insufficient lower extremities extensors muscle strength $(\mathrm{p}=0.039)$, slower COP moving rate $(\mathrm{p}<0.001)$ were also found. The asymmetry index has shown the discrepancy between normal subjects and OA knee patients which showed weight shifting to their sound side. There was a good correlation between the patient's Lequesne severity index and asymmetry index.

Conclusion: Objective assessment of the OA knee patient's functional performance reflects the patient's subjective assessment of their functional ability. OA knee patients shown a tendency of slow sit-to-stand movement, weakness lower extremities extensor muscle strength, poor posture control and weight shifting to sound side associated with reduced functional performance.

Keywords: Knee Osteoarthritis; Sit-To-Stand; Posture; Force Plate; Asymmetry Index

\section{Introduction}

Osteoarthritis $(\mathrm{OA})$ is the most common musculoskeletal disease in elderly population. Half of the people over fifty years old experience joint symptoms and have been found radiographic evidence of degenerative joint disease. According to the Taiwan National Health Insurance Statistics Yearbook in 2005 found that osteoarthritis-related outpatient medical costs is about 146 million. 
Knee is the most common site of OA and knee OA caused functional limitation and severe joint symptoms. Previous studies usually use radiographic evidence for diagnosis the severity of knee OA [1]. But the radiographic changes are not related to clinical symptoms and functional limitations [2]. Another way to evaluate knee OA severity can be quantified with either subjective questionnaires or performance measures [3-5]. Annoying joint symptoms such as pain, stiffness, and soreness made those patients difficult to stand up, squatting, and stair climbing. Patients learned to use compensate movement in daily life to readjust weight distribution which may reflect to functional performance [6]. In order to prevent joint symptoms while weight bearing, patients with knee OA shift their weight to unaffected limb. As time goes by, patients overused their unaffected limb and may cause bilateral knee OA [7].

Most study investigates gait deviation in such patients with severe knee $\mathrm{OA}$ to find the vicious cycle of weight shifting to unaffected limb. However, gait deviation did not be affected by mild OA, which will affect sit-to-stand [8]. In previous article Boonstra et al. has demonstrated that sit-to-stand testing can assess the knee function of TKA [9]. Therefore, it is reasonable for us to use the sitto-stand to see how the patient with knee OA will be, to compare the knee parameters in patient with different functional severity, and to see the compensatory strategy in those patients.

\section{Materials and Methods}

\section{Participants}

This is a case control study. Patients with knee osteoarthritis were recruited from the rehabilitation clinic at the Tri-service General Hospital from January 2008 to May 2009. The inclusion criteria are subjects were previously diagnosed with unilateral knee $\mathrm{OA}$ and had symptoms and signs consistent with knee OA and radiographic evidence. All patients were age between 50 and 88 years old and can follow order perform sit-to-stand without any assistance. Patients were excluded if they had neurological dysfunction, major musculoskeletal disease or had received lower extremity surgery, cognition deficit that cannot follow order. Based on Lequesne severity index, the patient group was further divided to mild (Grade 1), moderate (Grade 2), and severe functional limitation (Grade 3).All participants would be instructed for full assessment procedure by investigator and signed an Informed consent before experiment. Healthy controls without knee OA were also recruited for comparison. Our research project was sponsored by Project of Collaboration of Chi-Mei Medical Center and National Defense Medical Center with grant number (CMNDMC10212).

\section{Procedures}

According to patient's previous functional assessment of Lequesne severity index, all patients were classified to three functional limitation groups (mild, moderate, and severe). The Lequesne index is presented as a higher score indicating greater severity. The sit-to-stand testing was commenced by asking participants to sit on a custom-made device which was located above the force-plate. They were asked for barefoot and put both feet on the force plate (Figure 1). Our force-plate detected signals from 4 load-cells embed within the force plate and were connected to an amplifier transferring signals to computer for mathematical operations. All the original signal data were first input to LabVIEW 8.0 for immediately show up the participants' performance. Patients and controls could practice sit-to-stand twice on the force-plate then perform sit-to-stand for five times using as fast as possible they could. After abrupt stand-up, participants have to stand upright for 5 seconds. If participant cannot maintain steady standup for 5 second, investigator was waiting them to correct upright posture until steadiness. If patients cannot maintain steadiness to hold the security equipments, the data would be excluded.

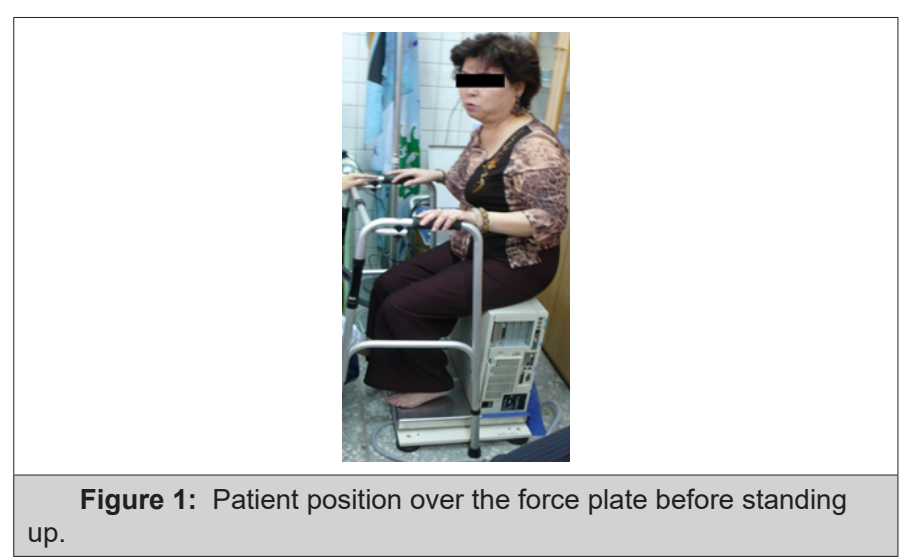

\section{Measurements}

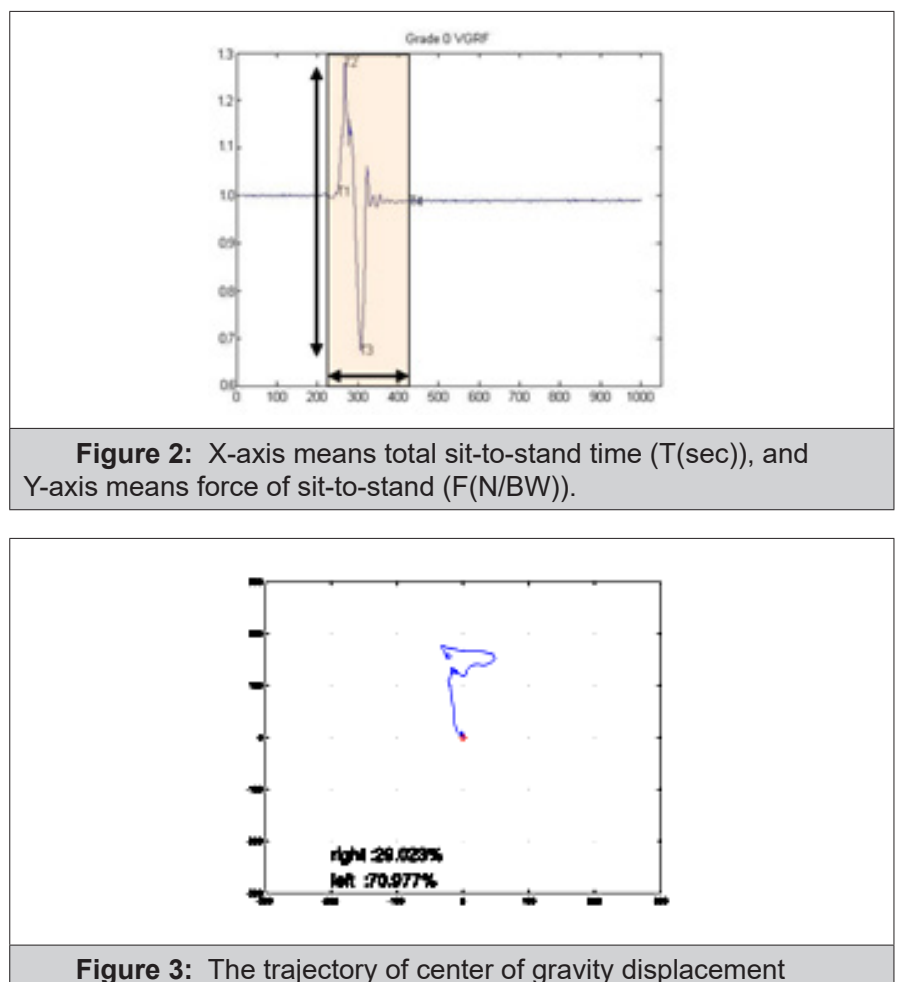

gure 3: The trajectory of center of gravity displacement

Our primary outcome included three time-force parameters: total sit-to-stand time (T, sec), the force of sit-to-stand (F, N/BW) (Figure 2), and the velocity of center of gravity displacement (Vcop, $\mathrm{cm} / \mathrm{sec}$ ) (Figure 3). The T means functional movement capacity which began from force plate detected the ground reaction force change more than $1 \%$ and end with the point when the vertical force oscillates inside a corridor of $2.5 \%$ plus/minus body weight [10]. 
The F means the lower extremities muscle groups strength while standing up which is ratio of ground reaction force difference and participant body weight. The Vcop means the motor control ability of sit-to-stand movement, and it comes from the total displacement divided to total sit-to-stand time. Center of gravity is substituted by center of pressure (COP) detected by load cell in force-plate. All the primary outcomes can be observed immediately on the computer monitor during every motion, so the investigator could encourage patient to perform a more rapid stand up movement as possible as they could. The reason for rapid motion is to obtain a real ground reaction force which represents the strength of lower extremities.

The secondary outcome was asymmetry index (ASI) which means the time difference of participant's center of gravity deviate to one side. ASI equaling 1 means absolute symmetry. ASI less than 1 means weight deviate to unaffected side. ASI more than 1 means participant's weight deviate to affected side. This study would like to figure out compensatory strategy form correlation of ASI and different functional limitation groups. ASI was calculated from the following equation:

$$
\mathrm{ASI}=\frac{X_{A s}-X_{S S}}{\left(X_{A S}+X_{\mathrm{sS}}\right)}+1 \quad \begin{aligned}
& X_{A s-\text { Weight deviate to affected (lef) side }} \\
& X_{S s-\text { Weight deviate to unaffected (right) side }}
\end{aligned}
$$

\section{Data analysis}

Then row signal data storage use text files including total force of the 4 load cells and the coordinate position of the force plate. The three time-force parameters were derived from combine signals using MATLAB 7.0 for further data calculation. The SPSS (16.0) was used for statistical analysis. One-way ANOVA was used to compare the difference of three parameters in four groups and used LSD for post hoc. The linear regression was used to analysis the relationship of Lequesne severity index score and the ASI score. A probability level less than 0.05 was indicative of statistical significance.

\section{Result [9a]}

Table 1: Subject Basic Data.

\begin{tabular}{|c|c|c|}
\hline Subject & Patients(n=27) & Controls(n=22) \\
\hline Gender (male: female) & $6: 21$ & $59.95 \pm 6.67$ \\
\hline Age $(\mathrm{yr})($ Mean \pm SD) & $65.00 \pm 9.18$ & $157.41 \pm 4.91$ \\
\hline Height $(\mathrm{cm})($ Mean \pm SD) & $155.74 \pm 6.88$ & $60.14 \pm 8.63$ \\
\hline Weight $(\mathrm{kg})($ Mean \pm SD) & $60.69 \pm 8.12$ & $24.33 \pm 3.75$ \\
\hline BMI $\left(\mathrm{kg} / \mathrm{m}^{2)}(\right.$ Mean \pm SD) & $25.23 \pm 3.74$ & Mean of Lequesne severity index \\
\hline Affected side (left: right) & $10: 17$ & 3.1 \\
\hline Osteoarthritis severity & Number & 10.92 \\
\hline Grade 1 & 10 & 16 \\
\hline Grade 2 & 12 & \\
\hline Grade 3 & 5 & \\
\hline
\end{tabular}

There was no significant difference in basic physical data between the OA patient group and control group (Table 1). The patient group was divided to Grade 1 for 10 subjects, Grade 2 for 13 subjects and Grade 3 for 5 subjects. Severe functional limitation group subjects were less than other group's subjects because they cannot perform sit-to-stand without assist or hand-hold. Health control group included 22 subjects.

\section{Primary Outcome}

The three time-force parameter all showed significant difference in four groups (Table 2). The post hoc for total sit-to-stand time and velocity of COP displacement showed Grade 0 and Grade 1 groups were different to Grade 2 and Grade 3 groups. (Figure 4-6) $(p<0.05)$ The force of sit-to-stand post hoc shown significant difference of Grade 0- Grade 2, and Grade 0- Grade 3 (Figure 5).

\begin{tabular}{|c|c|c|c|c|c|c|}
\hline \multicolumn{2}{|c|}{ Control } & \multicolumn{5}{|c|}{ Patient } \\
\hline & Grade $0(n=22)$ & Grade $1(n=10)$ & Grade $2(n=12)$ & Grade $3(n=5)$ & $F$ & $p$ \\
\hline $\mathbf{T}$ & $2.88 \pm 0.71$ & $2.73 \pm 0.72$ & $3.77 \pm 1.2$ & $4.78 \pm 2.48$ & 5.538 & $0.003^{*}$ \\
\hline (sec) & $2.88 \pm 0.71$ & $2.73 \pm 0.72$ & $3.77 \pm 1.2$ & $4.78 \pm 2.48$ & 5.538 & $0.003^{*}$ \\
\hline $\mathbf{F}$ & $5.78 \pm 1.88$ & $4.71 \pm 1.91$ & $4.06 \pm 2.89$ & $2.91 \pm 2.55$ & 3.022 & $0.039^{*}$ \\
\hline (N/BW) & $5.78 \pm 1.88$ & $4.71 \pm 1.91$ & $4.06 \pm 2.89$ & $2.91 \pm 2.55$ & 3.022 & $0.039^{*}$ \\
\hline Vcop & $191.57 \pm 51.49$ & $175.05 \pm 58.42$ & $129.88 \pm 33.7$ & $104.85 \pm 16.59$ & 7.414 & $0.000^{*}$ \\
\hline$(\mathrm{cm} / \mathrm{sec})$ & $191.57 \pm 51.49$ & $175.05 \pm 58.42$ & $129.88 \pm 33.7$ & $104.85 \pm 16.59$ & 7.414 & $0.000^{*}$ \\
\hline \multicolumn{7}{|c|}{${ }^{*} p<0.05 \mathrm{~T}$ : Total time (sec) } \\
\hline \multicolumn{7}{|c|}{ F: force of sit-to-stand (N/BW) } \\
\hline
\end{tabular}




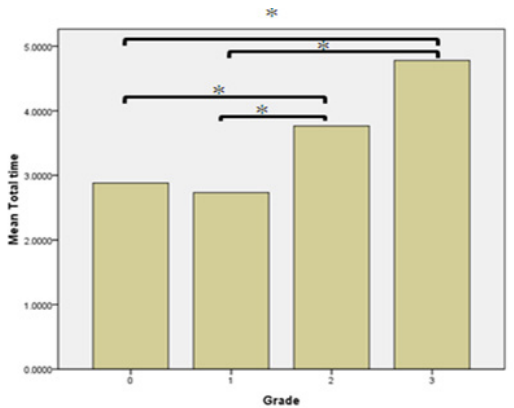

Figure 4: The bar figure of the post hoc of the total time in four group, ${ }^{*} p<0.05$.

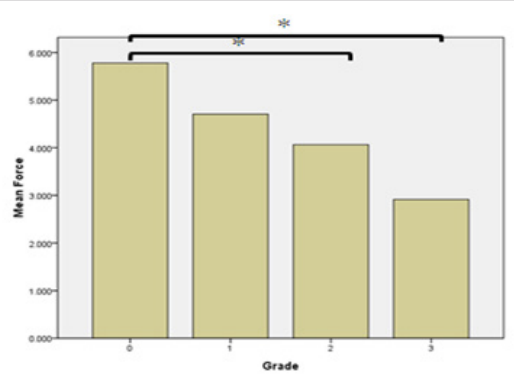

Figure 5: The bar figure of the post hoc of the force of sit-tostand in four group, ${ }^{*} p<0.05$.

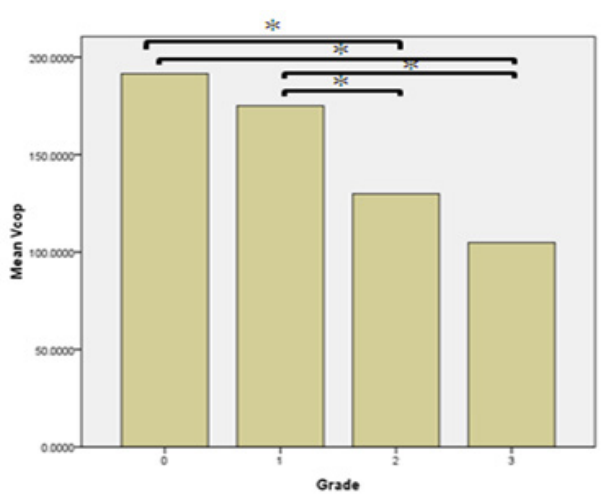

Figure 6: The bar figure of the post hoc of the velocity of COP displacement in four group, ${ }^{*} p<0.05$.

\section{Secondary Outcome}

The ASI showed non-significant difference between four groups (Table 3). But from Grade 0 to Grade 3, ASI had shown the tendency of less than 1. It means when knee is more serious, the patient tend to use their un-affected limb instead. The Lequesne severity index score and ASI score was related form statistic analysis. The liner regression result had shown significant relation between Lequesne severity index score and ASI score (Table 4).

Table 3: The ASI analysis.

\begin{tabular}{|c|c|c|c|c|c|c||}
\hline \multicolumn{5}{|c|}{ Control } & \multicolumn{5}{c|}{ Patient } \\
\hline & Grade 0 $(\mathrm{n}=22)$ & Grade 1 $(\mathrm{n}=10)$ & Grade 2(n=12) & Grade 3(n=5) & $\boldsymbol{F}$ & $\boldsymbol{p}$ \\
\hline ASI & $1.09 \pm 0.42$ & $0.82 \pm 0.51$ & $0.84 \pm 0.51$ & $0.77 \pm 0.23$ & 1.441 & 0.243 \\
\hline
\end{tabular}

Table 4: The relationship of Lequesne severity index and ASI.

\begin{tabular}{|c|c|c|c|c|c|}
\hline Model & Sum of Squares & df & Mean Square & $\mathbf{F}$ & $p$ \\
\hline Regression & 0.886 & 1 & 0.886 & \multirow{3}{*}{4.599} & \multirow{3}{*}{0.037} \\
\hline Residual & 9.05 & 47 & 0.193 & & \\
\hline Total & 9.936 & 48 & & & \\
\hline \multicolumn{3}{|c|}{ Unstandardized Coefficients } & \multicolumn{3}{|c|}{ Standardized Coefficients } \\
\hline Model & B & Std. Error & Beta & $\mathrm{t}$ & $p$ \\
\hline (Constant) & 1.054 & 0.082 & & 12.861 & 0 \\
\hline LSI & -0.023 & 0.011 & -0.299 & -2.145 & 0.037 \\
\hline \multicolumn{6}{|c|}{$\begin{array}{l}\quad * p<0.05 \\
\text { LSI: Lequesne severity index }\end{array}$} \\
\hline
\end{tabular}

\section{Discussion}

The purpose of this study is to compare the knee parameters in patient with different functional severity, and to see the compensatory strategy in those patients. The result of total sit-tostand time reflect that the moderate and severe functional limitation of knee OA patient take more time to stand up, which results were similar as those of Hurley et al. in the same groups [11]. The normal subjects and mild functional limitation group subjects showed no difference in sit-to-stand time, which responded the view of Terwee and colleagues whose review article suggested that functional limitation of mild knee OA patients acannot be distinguished by time measurement only [12]. In our study, functional limitation of the moderate group increased $130.9 \%$ total time in sit-to-stand testing, and the severe group increased $165.97 \%$ total time compared with control group, respectively. Similar tendency of increase sit-tostand time by the knee OA severity could be observed.

The force of sit-to-stand can be measured by force-plate and also reveals the subject's lower limbs strength, particularly in hip extensors and knee extensors. Previous studies revealed 
that knee OA patients decrease their quadriceps muscle strength while walking or other functional tasks $[11,13,14]$. In our study, the severe group showed minor peak ground reaction force and relatively more swing of the force during sit-to-stand. It means that severe knee OA subjects could not maintain strong muscle power and enough muscle strength in sit-to-stand task. The results in our study also showed that the difference of ground reaction force during sit-to-stand could reveal knee extensors strength in knee OA patients. Most researchers suggested that knee OA patients have weak knee extensors strength which is related to chronic pain and decreased function ability [15]. Our study verified the knee extensor weakness while performing sit-to-stand in moderate and severe knee OA patients.

The Vcop has been demonstrated as a parameter to assess the movement control ability [16]. Our results showed that the mild group had slower movement stability ability while stand up, and the COP displacement revealed the small difference between the mild and the control group by $8.62 \%$ decreased, even there was not significant difference. In our study we also found significant slower COP displacement speed in the moderate and severe groups. The COP displacement speed of moderate group was $32.2 \%$ slower than control, and the severe group was $45.27 \%$ slower than control. The deficit of movement control ability in knee OA patients has been considered as the quadriceps weakness and the proprioception loss $[17,18]$. Previous gait study using the lower extremity EMG also confirmed similar situation and suggested that co-contraction of the lower limbs muscle groups is the underlying reason to impair muscle control ability in knee OA patients [14]. The analysis of ASI did not show significant difference in four groups, which the reason might be the number of the severe group was far less than the other three groups, even the tendency of less than 1 indicates that patients would shift their weight to the unaffected side while stand up. The abnormal weight shifting in patient's movements should be the beginning of a vicious cycle to cause knee overloading in opposite side $[6,9,19]$. LiiKavainio et al. evaluated the symmetry of weightbearing functional task in normal subjects and non-symptomatic knee OA patients and found that patient had significant difference in descending stairs rather than in gait [8]. Our study also had the similar findings that the events of descending stair and sit-to-stand might stress the knee joint to induce patient's symptoms easily.

Previous studies used the time of repeated sit-to-stand to find the relationship between functional deteriorates and knee alignment changes in moderate and severe knee OA patients, but not in mild ones [20]. Another study used radiographic images to grade the severity of knee OA [21]. Both the methods failed to reflect patients' functional performance. Based on our result in significant relationship between Lequesne severity index score and ASI score, we objectively suggested using this sit-to-stand task to evaluate the functional disability or treatment effect in knee OA patients. Using ASI score we might detect the weight shifting changes and find out the corresponding of functional level in the early stage of knee OA. We assume that the relationship of functional limitation and knee biomechanical performance in sit-to-stand movement could provide clinical staff to identify the reality of knee disability in OA patients.
Using this simple task could also obtain the quantified score to understand the treatment effect. The limitation of our study was lack of sufficient participates of the four groups, in particular of the severe group. The small sample size of severe group was because those patients cannot perform sit-to-stand independently without hand-held or cannot maintain steadiness on the force-plate. And those data would be excluded after testing. For this reason, a long period of inclusion time will be needed for obtaining a large sample size in further study.

In conclusion, sit-to-stand performance could detect functional ability in knee OA patients rapidly and objectively. Functional limitation in moderate and severe knee $\mathrm{OA}$ patients showed slower movement time and poor movement control in sit-to-stand testing and had obvious knee extensor weakness as well. The more severe functional limitation patient is, the more weight shifting knee OA patient did.

\section{References}

1. Kellgren JH, Lawrence JS (1963) Atlas of standard radiographs: the epidemiology of chronic rheumatism. Vol 2. Oxford: Blackwell.

2. Kijowski R, Blankenbaker DG, Stanton PT, Fine JP, De Smet AA (2006) Radiographic findings of osteoarthritis versus arthroscopic findings of articular cartilage degeneration in the tibiofemoral joint. Radiology 239(3): 818-24.

3. Wewers ME, Lowe NK (1990) A critical review of visual analogue scales in the measurement of clinical phenomena. Research in Nursing and Health 13(4): 227-236.

4. Lequesne M, Mery C, Samson M, Gerard P (1987) Indexes of severity for osteoarthritis of the hip and knee: Validation-value in comparison with other assessment. Scandinavian journal of rheumatology. Supplement 65: 85-89.

5. Bellamy N, Buchanan WW, Goldsmith CH, Campbell J, Stitt LW (1988) Validation study of WOMAC: a health status instrument for measuring clinically important patient relevant outcomes to antirheumatic drug therapy in patients with osteoarthritis of the hip or knee. Journal of Rheumatology 15(12): 1833-1840.

6. McGibbon CA, Krebs DE (2002) Compensatory gait mechanics in patients with unilateral knee arthritis. Journal of Rheumatology 29(11): 2410-2419.

7. Mündermann A, Dyrby CO, Andriacchi TP (2005) Secondary gait changes in patient with medial compartment knee osteoarthritis. Arthritis \& Rheumatism 52(9): 2835-2844.

8. Liikavainio T, Isolehto J, Helminen HJ, perttunen J, lepola $\mathrm{V}$, et al. (2007) Loading and gait symmetry during level and stair walking in asymptomatic subjects with knee osteoarthritis: Importance of quadriceps femoris in reducing impact force during heel strike? The Knee 14(3): 231-238.

9. Boonstra MC, De Waal Malefijt MC, Verdonschot N (2008) How to quantify knee function after total knee arthroplasty? The knee 15(5): 390-395.

9a. Wang HY. Using sit-to-stand to assess the functional ability of patient with knee osteoarthritis. Master Thesis 2009.

10. Lindemann U, Claus H, Stuber M, Augat P, Muche R, et al. (2003) Measuring power during the sit-to-stand transfer. European journal of applied physiology 89(5): 466-470

11. Hurley MV, Scott DL, Rees J, Newham DJ (1997) Sensorimotor changes and functional performance in patients with knee osteoarthritis. Annals of the Rheumatic Diseases 56(11): 641-648. 
12. Terwee CB, Mokkink LB, Steultjens MP, Dekker J (2006) Performancebased methods for measuring the physical function of patients with osteoarthritis of the hip or knee: a systematic review of measurement properties. Rheumatology (Oxford) 45(7): 890-902.

13. Slemenda C, Brandt KD, Heilman DK, Mazzuca S, Braunstein EM, et al. (1997) Quadriceps weakness and osteoarthritis of the knee. Annals of internal medicine 127(2): 97-104.

14. Childs JD, Sparto PJ, Fitzgerald GK (2004) Alterations in lower extremity movement and muscle activation patterns in individuals with knee osteoarthritis. Clinical biomechanics (Bristol, Avon) 19(1): 44-9.

15. O’Reilly SC, Jones A, Muir KR, Doherty M (1998) Quadriceps weakness in knee osteoarthritis: the effect on pain and disability. Annals of the Rheumatic Diseases 57(10): 588-594.

16. Amiridis IG, Hatzitaki V, Arabatzi F (2003) Age-induced modifications of static postural control in humans. Neuroscience letters 350(3): 137-40.

17. Hassan BS, Mockett S, Doherty M (2001) Static postural sway, proprioception, and maximal voluntary quadriceps contraction in patients with knee osteoarthritis and normal control subjects. Annals of the Rheumatic Diseases 60: 612-618.

18. Lund H, Juul-Kristensen B, Hansen K, Christensen R, Christensen H, et al. (2008) Movement detection impaired in patients with knee osteoarthritis compared to healthy controls: a cross-sectional casecontrol study. Journal of musculoskeletal \& neuronal interactions 8(4): 391-400.

19. Shakoor N, Block JA, Shott S, Case JP (2002) Nonrandom evolution of endstage osteoarthritis of the lower limbs. Arthritis \& Rheumatism,46(12): 3185-3189.

20. Sharma L, Song J, Felson DT, Cahue S, Shamiyeh E, et al. (2001) The role of knee alignment in disease progression and functional decline in knee osteoarthritis. Journal of American Medical Association 286(2): 188195.

21. Barker K, Lamb SE, Toye F, Jackson S , Barrington S (2004) Association between radiographic joint space narrowing, function, pain and muscle power in severe osteoarthritis of the knee. Clinical Rehabilitation 18(7): 793-800. 